\title{
Promoter Analysis and Transcriptional Profiling of Ginkgo biloba 3- Hydroxy-3-Methylglutaryl Coenzyme A Reductase (GbHMGR) gene in Abiotic Stress Responses
}

\author{
Yongling $\mathrm{LIAO}^{1}$, Feng $\mathrm{XU}^{2 *}$, Xiaohua $\mathrm{HUANG}^{3}$, Weiwei $\mathrm{ZHANG}^{2}, \mathrm{Hua}^{\mathrm{C}} \mathrm{CHENG}^{3}$, \\ Linling $\mathrm{LI}^{3}$, Shuiyuan $\mathrm{CHENG}^{4}$, Yongbao SHEN ${ }^{1}$ \\ ${ }^{1}$ College of Forestry, Nanjing Forest University, Nanjing, 210037, China; liaoyongling@yeah.net; ybshen@nifu.com.cn \\ ${ }^{2}$ College of Horticulture and Gardening, Yangtze University, Jingzhou, 434025, China; xufeng198@126.com ("correspondingauthors); zww8312@163.com \\ ${ }^{3}$ Hubei Key Laboratory of Economic Forest Germplasm Improvement and Resources Comprehensive Utilization, Huanggang Normal University, Huanggang, 438000, \\ China; huangxiaohua87820@163.com;lilinling1437@126.com;harry1437@126.com \\ ${ }^{4}$ Schoolof Biology and PharmaceuticalEngineering,Wuhan Polytechnic University, Wuhan 430023, China;s_y_cheng@sina.com
}

\begin{abstract}
The terpene trilactones (TTLs) are believed to be important for the pharmacological properties of Ginkgo biloba leaves extract. 3-Hydroxy-3-methylglutaryl coenzyme A reductase (HMGR) is a critical enzyme involved in the biosynthetic pathway of TTLs. In this study, an 1.2-kb fragment of 5' flanking region of the HMGR gene (GbHMGR), was isolated from G. biloba by genome walking. Extensive sequence analysis revealed the presence of evolutionarily conserved and over-represented putative cis-acting elements in light-regulated transcription, hormone signaling (gibberellic acid, jasmonate and salicylic acid), elicitor and stress responses (cold/dehydration responses), and plant defense signaling (W-box/WRKY) that are common to the promoter region of GbHMGR. EMSA analysis suggested possible functionality of W-box in GbHMGR promoter region. The behavior of gene transcripts in ginkgo callus upon light, low temperature, MeJA and SA treatments further verified the regulatory function of $G b H M G R$ promoter. A significant positive relationship between gene expression level and total TTL contents suggested that GbHMGR might be one of key genes involved in TTL biosynthesis in G. biloba.
\end{abstract}

Keywords: EMSA, Ginkgo biloba, GbHMGR, promoter, terpene trilactones, expression level

\section{Introduction}

The maidenhair tree Ginkgo biloba L., known as living fossil, has undergone very little evolutionary changes over 200 million years and is considered to be native to China, Korea, and Japan (Singh et al., 2008). In recent years, standardized extracts of $G$. biloba leaves have been amongst the top-selling phytomedicines in the world (Gertz and Kiefer, 2004). Active compounds in G. biloba extract improve blood circulation, discourage clot formation, reinforce the walls of the capillaries and protect nerve cells from harm when deprived of oxygen (Mohanta et al., 2014). Flavonoids and terpene trilactones (TTLs) are believed to be associated with most of the pharmacological properties of G. biloba extracts. While flavonoids can be obtained from many other plants, ginkgolides and bilobalide, termed as TTLs, are unique components of G. biloba (Liao et al., 2011). Though TTLs is considered to play a key role in the active ingredients, the content of TTLs is very low, within $0.06 \%$ in dry leaves of $G$. biloba (van Beek and Montoro, 2009). Bioengineering is an ideal way to increase the content of TTLs, but which relies on the overall elucidation of the TTL biosynthetic pathway both at molecular genetics and biochemistry levels in G. biloba.

Terpenoids such as ginkgolides are biosynthesized from a universal 5-carbon building block Isopentenyl diphosphate (IPP) (Schwarz and Arigoni, 1999). IPP can be derived from two pathways: One is the classical cytosolic mevalonic acid (MVA) pathway and the other is the plastidial methylerythritol 4-phosphate (MEP) pathway, which is mevalonate independent. The MVA pathway in the cytosol, starting from 3 acetyl-CoA to finally yield IPP, is responsible for synthesizing sesquiterpenoids and sterols. The MEP pathway producing IPP and dimethylallyl diphosphate (DMAPP) from pyruvate and D-glyceraldehyde 3-phosphate (GAP) is mainly responsible for forming monoterpenoids, diterpenoids constituents. The classical cytosolic MVA pathway and the other is the plastidial MEP pathway, which 
26

is mevalonate independent (Lichtenthaler et al., 1997; Zeng et al., 2013). But the two pathways are not separated absolutely, in a certain extent, some unknown forms of crosstalk may occurred between them in some particular plants. For example, the biosynthetic precursor of ginkgolides derived from both MVA and MEP pathway in G. biloba (Schwarz, 1994; Schwarz and Arigoni, 1999). Therefore, numerous genes in both two pathways could contribute to the low level biosynthesis of ginkgolides. Up to now, many genes encoding enzymes involved in MVA and MEP pathway were isolated from G. biloba, including mevalonate diphosphate decarboxylase (MVD; Pang et al., 2006), 3-hydroxy3-methylglutaryl-Coenzyme A reductase (HMGR; Shen et al., 2006), 1-deoxy-D-xylulose 5-phosphate synthase (DXS; Gong et al., 2006; Kim et al., 2006a), 1-deoxy-D-xylulose 5-phosphate reductoisomerase (DXR; Gong et al., 2005; Kim et al., 2006a), 1hydroxy-2-methyl-2-(E)-butenyl-4-diphosphate synthase (HDS; Kim and Kim, 2010), 1-hydroxy-2-methyl-2-(E)-butenyl-4diphosphate reductase (IDS; Kim et al., 2008a), 2-C-methyl-Derythritiol 4-phosphate cytidyltransferase (MECT; Kim et al., 2006b), 2-C-methyl-D-erythritiol 2,4-cyclodiphosphate synthase (MECS; Kim et al., 2006c; Gao et al., 2006), and 4-(cytidine 5'diphospho)-2-C-methyl-D-erythritol kinase (CMK; Kim et al., 2008b).

3-hydroxy-3-methylglutaryl-Coenzyme A reductase (HMGR, EC: 1.1.1.34) catalyzes the conversion of HMG-CoA to MVA, which is a rate-limiting enzyme in isoprenoid biosynthesis via MVA pathway (Hunter, 2007; Buhaescu and Izzedine, 2007). In view of its significance in isoprenoid metabolism, genes encoding HMGR have been isolated and extensively characterized from many plants, including the medicinal plants Catharanthus roseus (Maldonado-Mendoza et al., 1992), Taxus media (Liao et al., 2004), Corylus avellana (Wang et al., 2007), Euphorbia pekinensis (Cao et al., 2010), and Salvia miltiorrhiza (Dai et al., 2011), Picrorbiza kurrooa (Singh et al., 2013), Withania somnifera (Akhtar et al., 2013), and Cyanotis arachnoidea (Wang et al., 2014). At present, a HMGR gene (GbHMGR) has been cloned from G. biloba (Shen et al., 2006). However, both expression pattern and the promoter region of $G b H M G R$ gene have not yet been clearly identified.

In the present study, we report the isolation and characterization of promoter region of GbHMGR gene to understand molecular regulation mechanism of GbHMGR expression underlying TTL biosynthesis in G. biloba. The transcript level of the GbHMGR and TTL content was examined after environmental stresses and plant hormone treatments. The correlation between transcript levels and TTL content in G. biloba callus further substantiated putative role of $G b H M G R$ gene in regulating TTL biosynthesis.

\section{Materials and methods}

\section{Plant materials and growth condition}

Seeds of ginkgo were harvested from 14-year-old grafts of $G$. biloba cultivar 'Jiafoshou' grown in Botanical Garden of Yangtze University, China in October, 2012. The seeds were used when the embryos were at the cotyledonary stage.

\section{Callusinduction of G. biloba and treatments}

The embryo-derived callus of $G$. biloba was induced using the method described by Zhang et al. (2011). The cultures were incubated in the light $\left(100 \mu \mathrm{mol} \mathrm{m} \mathrm{s}^{-1}\right)$ with a $16 / 8 \mathrm{~h} \mathrm{light/dark}$ photoperiod at $24 \pm 1{ }^{\circ} \mathrm{C}$ as the control. For dark treatment, fourweek-subcultured callus were covered with a cardboard box to keep in complete darkness and the samples were harvested at 24h from start of the treatment. For low temperature treatment, fourweek-subcultured callus were grown at $15 \pm 1^{\circ} \mathrm{C}$ and samples were harvested at $48 \mathrm{~h}$ after the treatment for analysis of gene transcription level and TTLs content. Methyl jasmonate (MeJA) and salicylic acid (SA) with the concentration $2.0 \mathrm{mM}$ were added to two-week callus cultured MS, and control cultures were untreated during cultivation. The samples was harvested and measured at 48 h after MeJA andSA treatments.

\section{Construction of genomic library and isolation of promoter region} of GbHMGR gene

Genomic DNA was extracted from ginkgo seed using modified CTAB method (Xu et al, 2008a). Ginkgo Genome walker libraries were constructed using the Genome Walker Universal Kit (Clontech, USA). To clone the promoter region of $G b H M G R$, two round PCR were performed using gene-specific primers (GbHMGRP1 and GbHMGRP2) that were designed according to the sequence of GbHMGR gDNA (AY741133; Pang et al., 2006), and the adapter primers (AP1 and AP2) of the kit. Their sequences were shown in Table 1. After the nested PCR was carried out, amplified fragments were cloned and then sequenced. The sequences that extended upstream of the gDNA of $G b H M G R$ were isolated as the 5 'upstream region of $G b H M G R$ gene and used for further analysis. The isolated 5'-upstream sequence was analyzed for the putative cis-acting regulatory elements using the PLACE (http://www.dna.affrc.go.jp/PLACE) and the Signal Scan Program PlantCARE (http://bioinformatics.psb.ugent.be/webtools/plantcare /html/) database.

\section{Expression of purification of GbWRKYsprotein in E. coli}

To obtain the WRKY protein, the open reading frame (ORF) of GbWRKY1 and GbWRKY2 of G. biloba (GbWRKY1 and GbWRKY2 Sequences in Supplemental Figure S1 and S2) was cloned into the expression vector pET-28a(+), yielding His-tagged GbWRKY1 and GbWRKY2. After sequence confirmation, the resulting recombinant plasmid was transferred into the $E$. coli strain BL21 (DE3) cells with heat shock method. A single colony of E. coli strain BL21 (DE3) cells harboring the plasmid pET28aGbWRKY1/GbWRKY2 was inoculated in LB medium at $37^{\circ} \mathrm{C}$ containing kanamycin $\left(50 \mathrm{mg} \mathrm{L}^{-1}\right)$, and was grown with $200 \mathrm{rpm}$ shaking at $37^{\circ} \mathrm{C}$ until the optical density (OD600) reached about 0.6. Expression of the recombinant protein was induced by adding isopropyl- $\beta$-D-1-thiogalactopyranoside (IPTG) and cells were harvested at $3 \mathrm{~h}$. The recombinant protein was extract and purified using Nickel-CL agarose affinity chromatography (Bangalore Genei, India) and used for electrophoretic mobility shift assay.

\section{Electrophoretic mobilityshift assay}

The W-box sequence in the promoter sequence of $G b H M G R$ was as TTTGAC using the PLACE and the Signal Scan Program PlantCARE database. Oligonucleotides of W-box sequence were synthesized and labeled with biotin (Shanghai Sangon Biotech Co., Ltd., China) for chemiluminescence using a LightShift Chemiluminescent EMSA Kit (Pierce Biotechnology, Thermo Fisher Scientific Inc., Rockford, USA). After labeling, 
complementary labeled strands were mixed together in an equimolar ratio and annealed at $25^{\circ} \mathrm{C}$ after denaturation at $90^{\circ} \mathrm{C}$. Gel mobility shift assays were performed by incubating $0.5 \mathrm{ng}$ of labeled probe with recombinant GbWRKYs protein and competing oligonuleotides in binding buffer $(10 \mathrm{mM}$ Tris- $\mathrm{HCl}$, $\mathrm{pH}$ 7.5, $50 \mathrm{mM} \mathrm{NaCl}, 1 \mathrm{mM}$ dithiothreitol, 1mM EDTA, 5\% glycerol, and $1 \mu \mathrm{g} / \mu \mathrm{L}$ poly $(\mathrm{dI} \bullet \mathrm{dC})$ ) at $25^{\circ} \mathrm{C}$ for $20 \mathrm{~min}$. Mixtures were size-fractionated on a non-denaturing $46 \%$ polyacrylamide gel followed by drying and transfer to nitrocellulose membranes and detection by streptavidin-HRP/chemiluminescence for biotin-labeled probes.

\section{Quantitative Real-Time PCR analysis of transcript levels}

The transcription levels of GbHMGR of G. biloba callus were determined at different stress or hormone treatments. RNA was isolated from the ginkgo callus at different treatments using CTAB methods (Cai et al., 2007). First-strand cDNA synthesis was performed in triplicate for each sample according to the instructions of the manufacturer (PrimeScript ${ }^{\mathrm{TM}}$ RT Reagent Kit, Dalian TaKaRa, China). Quantitative Real-Time PCR (qRTPCR) was carried out using a Applied Biosystems 7500 Real-Time PCR System with SYBR Premix Ex Taq ${ }^{\text {tx }}$ II Kit (Dalian TaKaRa, China) according to the protocol of the manufacturer. The primers of GbHMGR and G. biloba house-keeping gene $18 S$ (GenBank accession no. D16448) for qRT-PCR are listed in Table 1. Reactions were performed in triplicate using $10 \mu \mathrm{L}$ of SYBRPremix Ex Taq II, $0.8 \mu \mathrm{L}$ of each primer, $0.4 \mu \mathrm{L}$ ROX Reference Dye II, $2 \mu \mathrm{L}$ of diluted cDNA, and nuclease free water to a final volume of $20 \mu \mathrm{L}$. The PCR reaction conditions were preincubated at $95^{\circ} \mathrm{C}$ for $30 \mathrm{~s}$, followed by 30 cycles of amplification $\left(95^{\circ} \mathrm{C}\right.$ for $5 \mathrm{~s}, 60^{\circ} \mathrm{C}$ for $\left.34 \mathrm{~s}\right)$, with melt curve stage $\left(95^{\circ} \mathrm{C}\right.$ for $15 \mathrm{~s}$, $60^{\circ} \mathrm{C}$ for $1 \mathrm{~min}$, and $95^{\circ} \mathrm{C}$ for $15 \mathrm{~s}$ ). Fluorescence was measured at the end of each annealing step. Raw data were analyzed with Applied Biosystems 7500 software, and expression was normalized to $18 S$ gene to minimize the variation in the cDNA template levels. Real-time PCR data were technically replicated with error bars, representing mean $\pm S E(n=3)$.

\section{Extraction and determination of TTLs}

Ginkgolide A (GA), ginkgolide B (GB), ginkgolide C (GC) and bilobalide $(\mathrm{BB})$ were extracted and determined using gas chromatography with a wide bore capillary column (Liao et al., 2008). The content of TTLs was the sum of the contents of GA, $\mathrm{GB}, \mathrm{GC}$, and BB and expressed as $\mu \mathrm{g} \cdot \mathrm{g}^{-1} \mathrm{DW}$. All the tests were carried out in triplicate, and data represent the means \pm standard errors (SE).

\section{Statistic analysis}

Data were analyzed with one-way ANOVA using SPSS 11.0 (SPSS Inc., Chicago, Illinois) for Windows and means were compared with Duncan's multiple range test at $\mathrm{P}<0.05$.

\section{Results and discussions}

\section{Cloning and analysis of GbHMGR promoter}

The promoter sequence of $G b H M G R$ was obtained by constructing a Genomic Walker DNA library from $G$. biloba leaves after 2 rounds of nested PCR using chromosome walking techniques. The promoter sequence of $G b H M G R$ gene was 1,264 bp in length (Fig. 1). The cis-acting elements of GbHMGR promoter was predicted using PlantCARE and PLACE database. Various cis-acting elements, along with their functions and location in the promoter of GbHMGR, are shown in Table 2 and Fig. 2. We found that the GbHMGR promoter sequence contains many important cis-regulatory elements such as TATA boxes, CAAT boxes, ACGTT boxes, W boxes, E boxes, etc. Specifically, in silico analysis showed that one TATA boxes are present within the promoter region of GbHMGR, at position 1233 , i.e. 32 bp upstream of the transcription start site. The TATA box is necessary in promote gene transcription by combination to RNA polymerase II, and affects the rate of transcription (Smale and Kadonaga, 2003). Another conserved eukaryotic cis-element, CAAT boxes, were also observed at position 1109 , i.e. $124 \mathrm{bp}$ upstream from TATA box. The CAAT box controls the transcription initiation frequency and impacts conversion rates of target genes (Edwards et al., 1998). The changes in these basic elements within the promoter region will greatly affect the level of transcription of target genes. In addition to these essential cis-acting elements, other corresponding cis-element with roles in regulation of gene expression in the promoter region of $G b H M G R$ were also predicted and listed in Table 2. These include cis-elements associated with hormone regulations and found in other plant gene promoters, in detail including one ABRERATCAL motif function as $\mathrm{Ca}^{2+}$-responsive element (Kaplan et al., 2006), one CATATGGMSAUR element involved in the induction of gene by Auxin (Xu et al., 1997), two Arabidopsis Response Regulator 1 type B (ARR1AT) transcription factor recognition sequences involved in cytokinin signaling and two sequences critical for cytokinin-enhanced binding (Sakai et al., 2000), one MYBGAHV element and three GT1 motifs known as to be GA and SA-responsive elements (Gubler et al.,1999; Zhou, 1999), respectively. We also found the presence of eight Dof (DNA binding with one finger) transcription factor recognition core sequences, which could be involved in auxin, jasmonate or ethylene responsiveness as previously reporter (Baumann et al., 1999; Yanagisawa and Schmidt, 1999; Nakano et al., 2006). In addition, the GbHMGR promoter contains cis-elements previously associated with low-temperature and light responsiveness, including three GT1 motifs (Zhou, 1999), one CRTDREHVCBF2 element (Xue, 2003) and one T box (Chan et al., 2001). Interestingly, we also found one MYB-box (MYB1AT), three W-box and four E-boxes in promoter region of GbHMGR. The W-box and MYB-box have shown to be WRKY (Eulgem et al., 2000) and MYB (Abe et al., 2003) transcription factor binding sites, respectively, involved in plant defense signaling. The consensus E-box sequence has been shown to be recognized by the basic helix-loop-helix (bHLH) proteins and involved in light regulation (Hartmann et al., 2005). The bioinformatic analysis also revealed the presence of root motif for root specific expression in GbHMGR promoter region (Elmayan and Tepfer, 1995). Previous study has shown that the transcript of $G b H M G R$ was present specifically in roots (Shen et al., 2006). Three ROOT-motif elements (positions 184, 407 and 753) were identified as root-responsive ciselements, which were consistent with the expression pattern of GbHMGR gene. Based on the predictive identification of putative cis-acting element, it could be hypothesized that the transcriptional activity of the GbHMGR promoter is regulated by different signals. 
28

Table 1. Primer sequences used in the experiment

\begin{tabular}{lcc}
\hline \multicolumn{1}{c}{ Name } & Sequence $\left(5^{\prime}-3^{\prime}\right)$ & Description \\
\hline AP1 & GTAATACGACTCACTATAGGGC & Reverse primer for promoter amplification, outer \\
AP2 & ACTATAGGGCACGCGTGGT & Reverse primer for promoter amplification, nested \\
GbHMGRP1 & ACGCCCTACTCTTCAACTCCTCCCTTCT & Reverse primer for promoter amplification, outer \\
GbHMGRP2 & ATACGAATGAAGCCAACTGAGCCACGAT & Reverse primer for promoter amplification, nested \\
HMGRFP & TCTTTGTCATGTTTTTCAGC & Gene specific primer for qRT-PCR, forward \\
HMGRRP & AGTTTCTCTTTCTCTCTCGC & Gene specific primer for qRT-PCR, reverse \\
18SFP & ATAACAATACTGGGCTCATCG & Gene specific primer for qRT-PCR, forward \\
18SRP & TTCGCAGTGGTTCGTCTTTC & Gene specific primer for qRT-PCR, reverse \\
\hline
\end{tabular}

Table 2. Putative cis-acting regulatory elements identified in the promoter of GbHMGR using PLACE and PlantCARE database

\begin{tabular}{|c|c|c|c|}
\hline Factor or Site Name & Position & Signal Sequence & Expected function \\
\hline ABRERATCAL & 7 & MACGYGB & $\mathrm{Ca}^{2+}$-responsive up-regulated genes \\
\hline CRTDREHVCBF2 & 15 & GTCGAC & Low-temperature responsive \\
\hline CGACGOSAMY3 & 17,1218 & CGACG & May function as a coupling element for the $\mathrm{G}$ box element \\
\hline DOFCOREZM & $\begin{array}{c}41,140,267,304,442,817 \\
839,1019\end{array}$ & AAAG & $\begin{array}{l}\text { Dof } 1 \text { and Dof } 2 \text { transcription factors are associated with } \\
\text { expression of multiple genes involved in carbon metabolism }\end{array}$ \\
\hline GT1 motif & $76,209,962$ & GRWAAW & $\begin{array}{l}\text { Consensus GT-1 binding site in many light-regulated genes and } \\
\text { influence the level of of SA-inducible gene expression }\end{array}$ \\
\hline ROOTMOTIFTAPOX1 & $184,407,753$ & ATATT & Root specific expression \\
\hline $\mathrm{EBOX}$ & $219,278,601,862$ & CANNTG & $\begin{array}{c}\text { Cis-element binding BHLH factor involved in light } \\
\text { responsiveness and tissue-specific }\end{array}$ \\
\hline T Box & 241 & ACTTTG & $\begin{array}{c}\begin{array}{c}\text { Mutations in the 'T box' resulted in reductions of light-activated } \\
\text { gene transcription }\end{array}\end{array}$ \\
\hline W Box & $282,483,855$ & TGAC & WRKY binding site, involved in many physiological processes \\
\hline MYB1AT & 347 & WAACCA & MYB recognition site involved in dehydration-responsive \\
\hline TATA Box & 1233 & TATAA & Common cis-acting element in promoter and enhancer regions \\
\hline CURECORECR & $334,383,1238$ & GTAC & Copper-responsive element \\
\hline CAAT Box & 1109 & CAAT & Common cis-acting element in promoter and enhancer regions \\
\hline NTBBF1ARROLB & $474,993,1127$ & ACTTTA & Required for tissue-specific expression and auxin induction; \\
\hline CATATGGMSAUR & 601 & CATATG & Auxin-responsive \\
\hline MYBGAHV & 737 & TAACAAA & $\begin{array}{c}\text { Central element of gibberellin (GA) response complex (GARC) } \\
\text { in high-pI alpha-amylase gene }\end{array}$ \\
\hline ARR1AT & $1172,81,448$ & NGATT & ARR1-binding element \\
\hline ACGTTBOX & 1073,1207 & AACGTT & One of ACGT elements \\
\hline
\end{tabular}

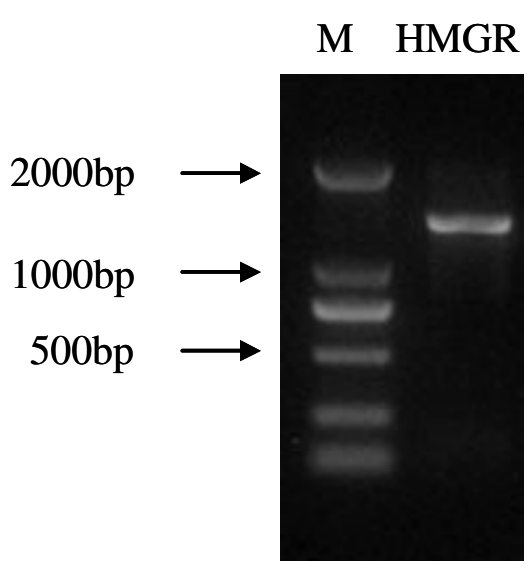

Fig. 1. Promoter sequences of GbHMGR. Lane M, DNA marker DL2000; HMGR, PCR product of the GbHMGR gene promoter

Comparison of the known promoter sequences of the genes such as GbDXS and GbGGPPS (Xu et al., 2013), involved in TTL biosynthetic pathway in $G$. biloba, reveals that cis-elements binding Dof proteins. Dof proteins are plant-specific transcription factors with a highly conserved DNA-binding domain, which presumably induces a single C2-C2 zinc finger. Zinc finger proteins like members of the transcription factor IIIA zinc finger protein family (ZCTs) have been previously isolated in medicinal plant Catharanthus roseus and were shown to act as transcriptional repressors of TDC and STR promoter activity in the regulation of induced terpenoid metabolism (Pauw et al., 2004). Dof transcription factors have been suggested to participate in the regulation of vital processes exclusive to plants such as photosynthetic carbon assimilation, light-regulated gene expression, accumulation of seed-storage proteins, germination, dormancy and response to phytohormones (Yanagisawa 2004). The relative abundance of Dof binding sites in $G b H M G R$ promoter suggest that this specific protein could play a significant role of TTL gene expression in G. biloba.

\section{GbWRKYs bind with the $W$-box sequence of GbHMGR} promoter

Several reports have shown that WRKY proteins regulate the expression of gene involved in terpenoid biosynthesis by combining the W-box, and W-box was found in present in gene promoters related with MEP pathway such as LPS (Kim et al., 2012), IDSs (Kang et al., 2013), GbDXS and GbGGPPS (Xu et al., 2013). The W-box sequences predicted as TTTGAC were 


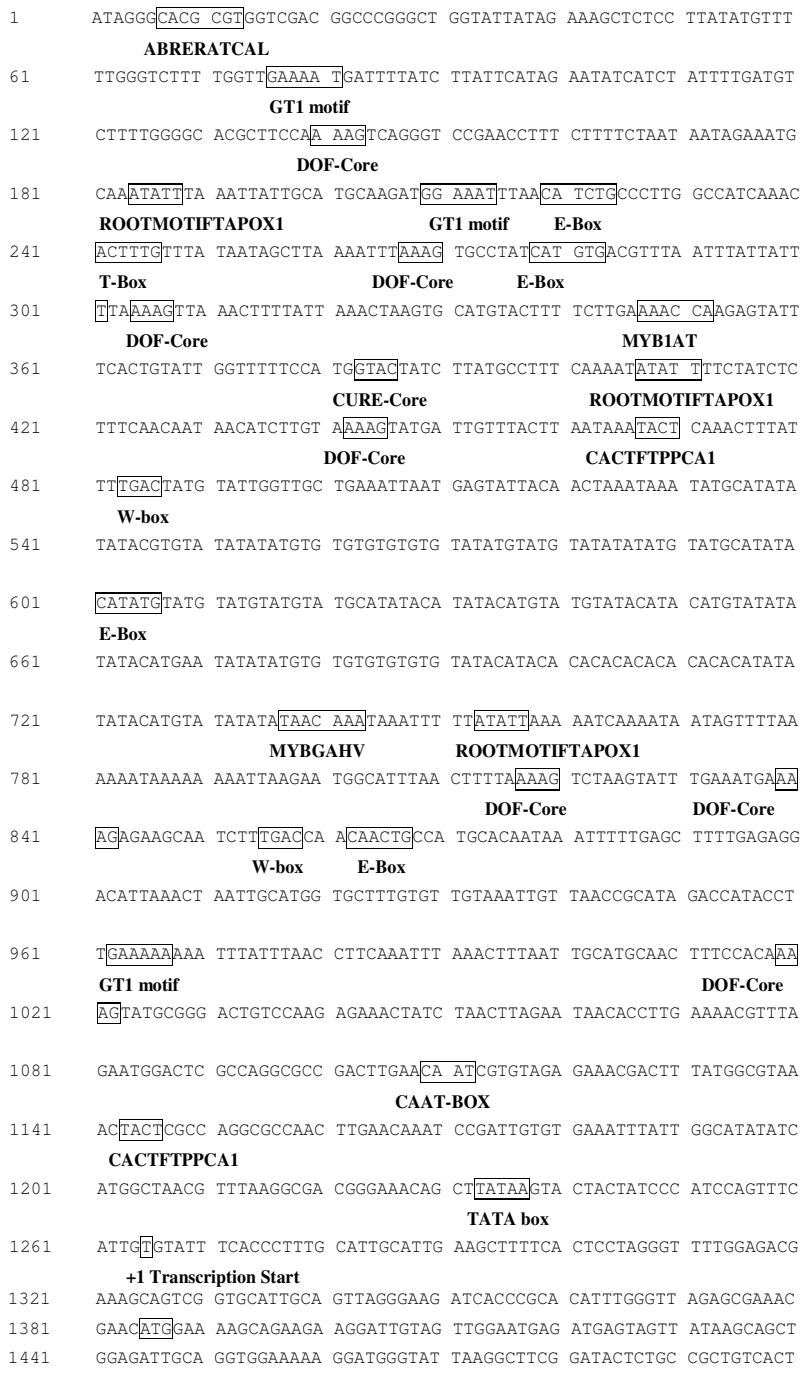

Fig. 2. The sequence of the GbHMGR promoter region. The regulatory elements in the $G b H M G R$ promoter are boxed
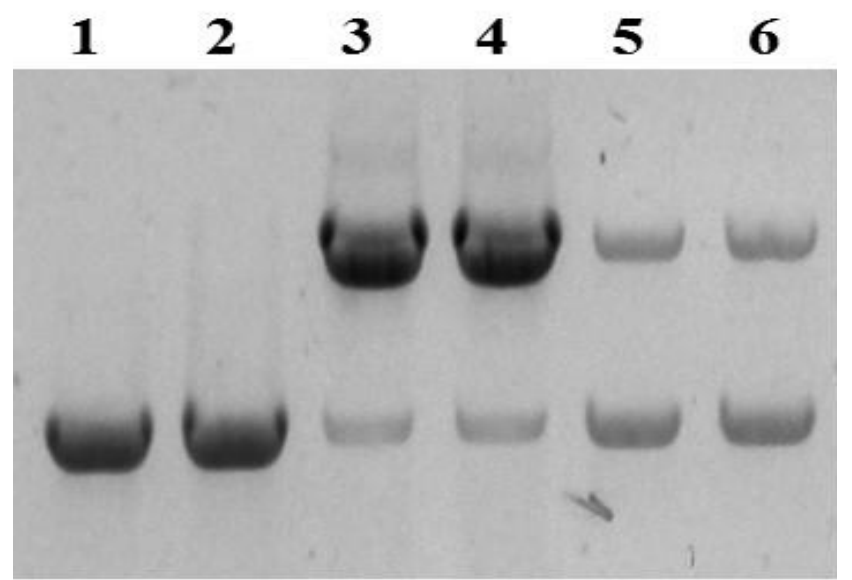

Fig. 3. Gel mobility shift assays for W-box of the GbHMGR promoter. 1. E. coli protein mixed biotin labeled W-box probe; 2, pET28a protein mixed biotin labeled W-box probe; 3, 4, the recombinant GbWRKY1 and GbWRKY2 mixed biotin labeled W-box probe, respectively; 5,6, GbWRKY1 and GbWRKY2 mixed biotin labeled and unlabeled W-box probes also found in the promoter sequence of $G b H M G R$. Our group have cloned 31 WRKY genes based $G$. biloba transcriptome data. Our unpublished results indicated two WRKY genes $G b W R K Y 1$ and GbWRKY2 (ORF sequences in Supplemental Fig. S1. and S2.) might be involved in TTL biosynthetic pathway. GbWRKY1 and GbWRKY2 were expressed in E. coli, respectively. Upon induction by IPTG, GbWRKY1 (Supplemental Fig. S3, lans 2 and 3) and GbWRKY2 (Supplemental Fig. S3, lan 4 and 5) was expressed as a major protein product in the total celluar soluble protein. The molecular weight of the expressed recombinant GbWRKY1 and GbWRKY2 proteins was estimated to be about 36.7 and 39.7 $\mathrm{kDa}$ with the His-tag, respectively, the size of which were in good agreement with that predicted through bioinformatics. The interaction between GbWRKY1 and GbWRKY2 with GbHMGR promoter sequence was assayed with EMSA. No binding bands were detected with crude proteins of $E$. coli without or with empty vector pET28a (Fig. 3, lans 1 and 2). GbWRKY1 and GbWRKY2 specifically bind with the W-box sequence, and unlabeled probes inhibit the binding (Fig. 3). These results confirmed that GbWRKY proteins could combine to the W-box sequence of GbHMGR gene which is the target gene of WRKY protein. Some studies have also reported the WRKY proteins participated in the control of sesquiterpene and enzylisoquinoline alkaloid biosynthesis and their transcriptional induction by methyljasmonate (Xu et al., 2004; Kato et al., 2007; Ma et al., 2009). Data on EMSA suggested that GbWRKY1 and GbWRKY2 might regulate TTL accumulation through regulating the transcript level of target gene GbHMGR in G. biloba.

\section{Effect light and low temperature on expression of GbHMGR} and TTL content

As shown in Fig. 4A, GbHMGR expression level of ginkgo callus was significantly $(P<0.05)$ higher by $281.3 \%$ in light as compared to those under dark after $24 \mathrm{~h}$ treatment. Also, total TTL content of ginkgo callus increased by $18.0 \%$ in light as compared to the callus maintained under dark after 24 treatment (Fig. 4B). The above results implied that dark conditions might starve the plants and it is likely that gene expression and TTL content in dark could be a reflection of the effect of carbon limitation. Light would affect carbon pool through photosynthesis and the role of carbon pool in regulating secondary metabolites has been shown in Pinus sylvestris (Heyworth et al., 1998) and Hypericum perforatum (Mosaleeyanon et al., 2005). Our previous work also demonstrated that chlorocholine chloride induced the biosynthesis of TTL and expression of key genes related with ginkgolide biosynthesis by promoting the photosynthesis and carbon pool (Xu et al., 2011). Also, the possibility exists that light modulated gene expression independent of carbon pool (Fey et al., 2005). Moreover, GbHMGR expression was in agreement with the promoter data wherein the motifs (e.g. GT1 motif, T box, and E box) for light were present. The role of light in modulating a range of terpenoids and the corresponding transcripts has been documented, but there is no universal behavior and it varies depending upon the metabolite type and the plant species. The same gene, $H M G R$ for example, it stimulated by light in Triticum aestivum (Aoyagi et al., 1993) and potato (Korth et al., 2000). Effect of light on the activity of $H M G R$ promoter has been reported that explained the light mediated alteration in 
30

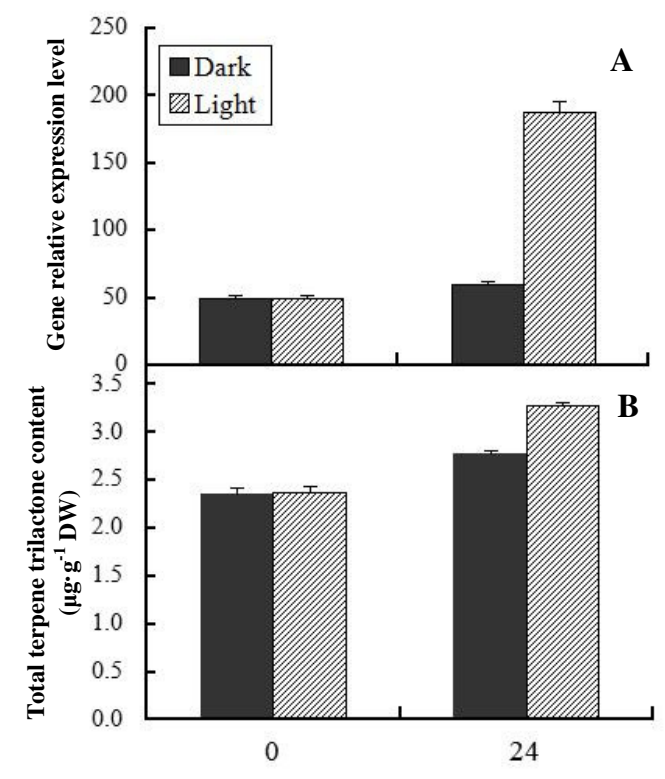

Hours after Treatment

Fig. 4. Effect of light on expression level of $G b H M G R(\mathrm{~A})$ and the content of total terpene trilactones (B). Total RNA samples were isolated from ginkgo callus and subjected to realtime PCR. Values are the mean of six callus samples and bars represent standard errors.

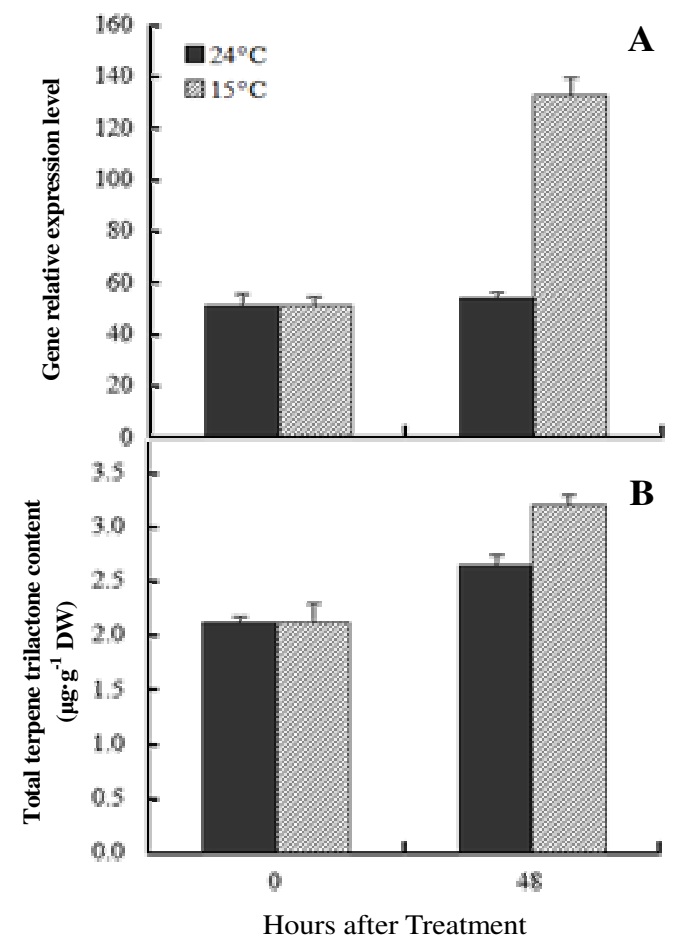

Fig. 5. Effect of low temperature on expression level of GbHMGR (A) and the content of total terpene trilactones (B). Total RNA samples were isolated from ginkgo callus and subjected to real-time PCR. Values are the mean of six callus samples and bars represent standard errors
$H M G R$ transcripts (Learned and Connolly, 1997; Kawoosa et al., 2010).

In the case of low temperature, both expression of GbHMGR and total TTL content were significantly $(P<0.05)$ higher by $143.1 \%$ and $21.0 \%$, respectively at $15^{\circ} \mathrm{C}$ as compared to those at $24^{\circ} \mathrm{C}$ (Fig. $5 \mathrm{~A}$ and $\mathrm{B}$ ). The up-regulation of $G b H M G R$ by low temperature is expected because one low temperature responsive motif was identified in $G b H M G R$ promoter region. Picrorhiza $H M G R$ gene has also been reported to be up-regulated by low temperature due to the motif for low temperature presence in the promoter region of this gene. Likewise, our previous work also found that low temperature could up-regulated the genes such as GbPAL (Xu et al., 2008a), GbANS (Xu et al., 2008b), and GbFLS (Xu et al., 2012) involved in flavonoid biosynthesis in G. biloba. Taken together, it can be suggested that secondary metabolite production can be induced by low temperature under the temperature condition of satisfy the growth of ginkgo callus.

Soitamo et al. (2008) showed that light at low temperature induced expression genes involved in synthesis of phenylpropanoids, carotenoids, and terpenoids. In Arabidopsis, light-dependent flavonoid (Fuglevand et al., 1996) and phenylpropanoid (Hemm et al., 2004) biosynthesis has been attributed to upregulation of relevant genes at transcript level, and the involvement of the enhancement of primary metabolite production. Low temperature mediated increase in secondary metabolites in Arabidopsis has mainly been attributed to the transcriptional upregulation of genes of secondary metabolism, which in turn, has been suggested due to the over-expression of relevant transcription factors at low temperature (Hannah et al., 2005). It also possible that upregulation of $G b H M G R$ by light and low temperature increased carbon partitioning towards terpenoid metabolism resulting in higher TTL content in $G$. biloba.

\section{Effect MeJA and SA on expression of GbHMGR and TTL content}

Various signaling molecules that interact with their cognate receptors in the plant plasma memberane activate specific genes, which are responsible for the synthesis of alkaloids among secondary plant metabolites (Menke et al., 1999). Among the various signaling molecules, the elicitors MeJA and SA are thought to activate signal transduction pathways that stimulate expression of the enzymes, which form defense compounds such as terpenoids (Martin et al., 2003; Pu et al., 2009). Thus, this paper study the effects of MeJA and SA on expression of GbHMGR and accumulation of TTLs in ginkgo callus. As shown in Fig. 6, MeJA significantly stimulated GbHMGR expression and total TTL content by $86.5 \%$ and $23.9 \%$, respectively, after $48 \mathrm{~h}$ treatment. Similarly, SA treatment at $48 \mathrm{~h}$ caused increasing expression level of $G b H M G R$ expression and total TTL content by $208.6 \%$ and $16.5 \%$ (Fig. 7 ), respectively. Response of GbHMGR expression to MeJA and SA could provide clue to the function of the enzymes. MeJA- and SAresponsive sites are present in the promoter sequence of GbHMGR (Fig. 2 and Table 2). Production of MeJA and SA is involved in plant defense against biotic stresses such as herbivore and pathogen attacks (Robert-Seilaniantz et al., 2011). MeJA and SA interact antagonistically against each other to induce transcription of defense-related genes (Koornneef et al., 2008). 


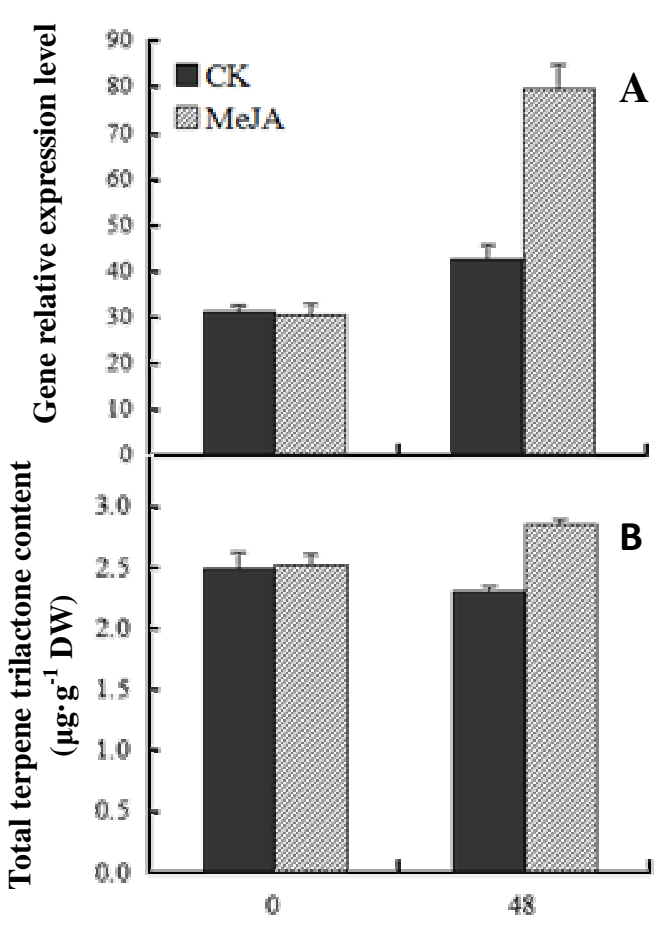

Hours after Treatment

Fig. 6. Effect of methyl jasmonate on expression level of $G b H M G R$ (A) and the content of total terpene trilactones (B). Total RNA samples were isolated from ginkgo callus and subjected to real-time PCR. Values are the mean of six callus samples and bars represent standard errors

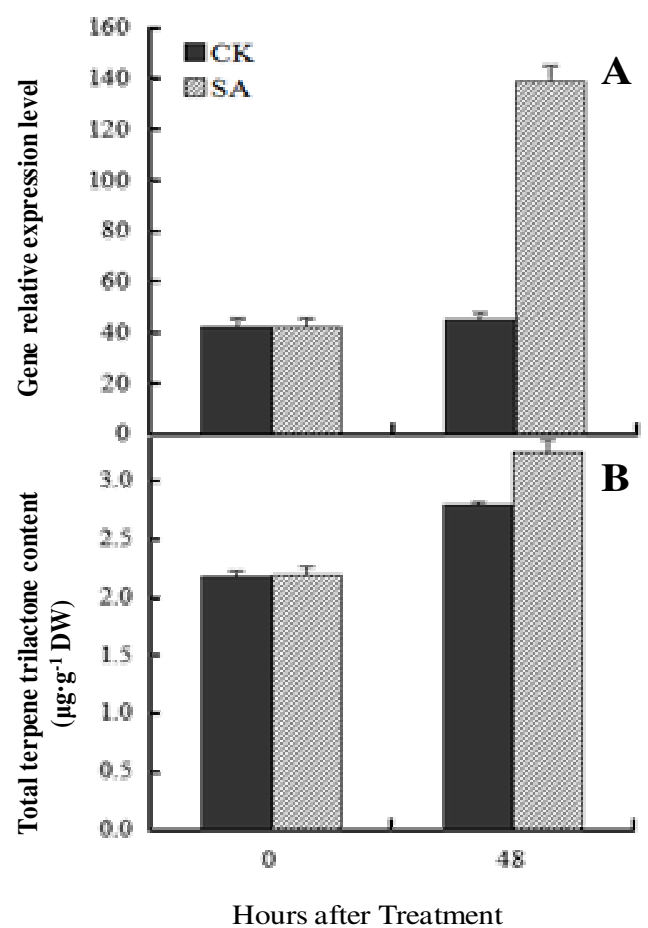

Fig. 7. Effect of salicylic acid on expression level of $G b H M G R$ (A) and the content of total terpene trilactones (B). Total RNA samples were isolated from ginkgo callus and subjected to real-time PCR. Values are the mean of six callus samples and bars represent standard errors

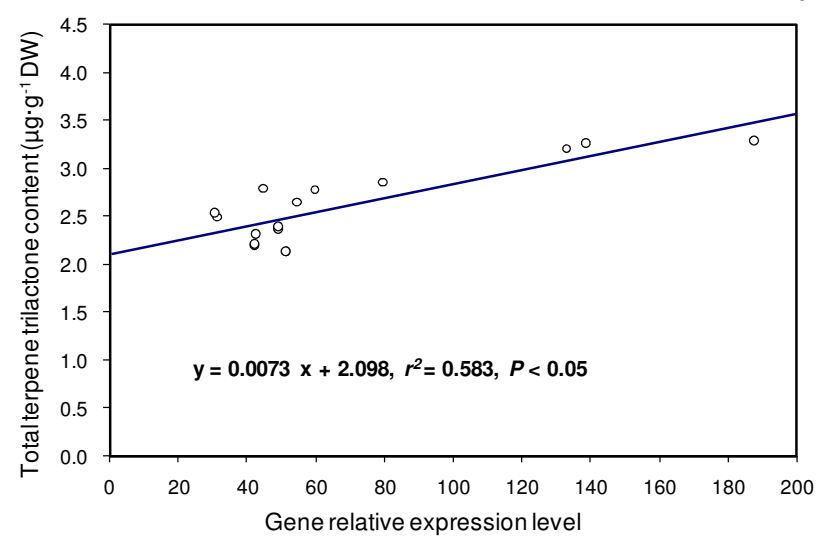

Fig. 8. Relationship between the content of total terpene trilactones and expression level of GbHMGR in Ginkgo biloba $(n=16)$

The HMGR genes were also induced by MeJA and SA in Solanum tuberosum (Choi et al., 1994), Brassica juncea (Alex et al., 2000), Artemisia annua (Pu et al., 2009), and S. miltiorrbiza (Liao et al., 2009; Dai et al., 2011). C. arachnoidea (Wang et al., 2014). The up-regulation of $H M G R$ expression by MeJA and $\mathrm{SA}$ is of particular interest because of the possible limiting role of this enzyme in terpenoid synthesis. However, MeJA and SA are not specific inducer of the $G b H M G R$ gene. They also induces other genes contributing to terpenoid biosynthesis. In G. biloba, genes IDS, DXS, CMK have also been reported to be positively responsive toward MeJA and SA treatment (Gong et al., 2006; Kim et al., 2008a, b). They suggested that the positive response implied the involvement of the gene in ginkgolide biosynthesis. Therefore, it is considered that the increased content of TTLs by MeJA or SA in present study is due to an integrated effect on a cluster of genes related to TTL biosynthesis. Further work will be required to study the effect of MeJA and SA on expression of more related genes involved in TTL biosynthesis.

\section{Relationship between GbHMGR expression and TTL} accumulation

To further study relationship between of GbHMGR expression and TTL accumulation. We performed linear regression analysis of gene expression level and total TTL content data in ginkgo callus subjected to the light, low temperature, MeJA and SA treatments. The results showed that the relationship between total TTL content (y) and GbHMGR expression level $(\mathrm{x})$ was significantly positively linearly correlated (Fig. 8), with a correlation coefficent is $r^{2}=0.583$, and a linear curve equation represented by $\mathrm{y}=(0.0073 \pm 0.0014) \mathrm{x}+(2.098$ \pm 0.1112 ). These results indicated that the GbHMGR gene is responsible for the TTL accumulation and might played a crucial role in TTL biosynthesis.

\section{Conclusions}

In summary, the present study isolated and characterized the MVA pathway GbHMGR gene promoter from G. biloba. Functional and bioinformatic analyses revealed that the GbHMGR promoter contains a number of cis-motifs that could bind transcription factors involved in regulation of TTLs. EMSA analysis suggested possible functionality of W- 
32

box in GbHMGR promoter region. The behavior of gene transcripts in ginkgo callus upon light, low temperature, $\mathrm{MeJA}$ and SA treatments further verified the regulatory function of $G b H M G R$ promoter. A positive relationship between gene expression level and total TTL contents implied that $G b H M G R$ might be one of key genes involved in TTL biosynthesis in G. biloba. Studies on gene promoters in terpenoid biosynthetic pathway constitute a valuable approach to identify new regulatory factors and/or families that could controlled the TTL biosynthesis in G. biloba cell culture.

\section{Acknowledgments}

This work was supported by National Natural Science Foundation of China (31370680, and 31400556), the Natural Science Foundation of Hubei Province (2013CFA039), the Foundation for Innovative Research Groups of Natural Science of Hubei Province (2011CDA117), and International Science and Technology Cooperation Project of Hubei Province (2013BHE029 and 2013BHE039), and the Graduate Innovative Project of Jiangsu Province (2013CXZZ13-D551).

\section{References}

Abe H, Urao T, Ito T, Seki M, Shinozaki K, Yamaguchi-Shinozaki K (2003). Arabidopsis AtMYC2 (bHLH) and AtMYB2 (MYB) function as transcriptional activators in abscisic acid signaling. Plant Cell 15:63-78.

Akhtar N, Gupta P, Sangwan NS, Sangwan RS, Trivedi PK (2013). Cloning and functional characterization of 3-hydroxy-3methylglutaryl coenzyme A reductase gene from Withania somnifera: an important medicinal plant. Protoplasma 250:613622.

Alex D, Bach TJ, Chye ML (2000). Expression of Brassica juncea 3hydroxy-3-methylglutaryl CoA synthase is developmentally regulated and stress-responsive. Plant J 22:415-426.

Aoyagi K, Beyou A, Moon K, Fang L, Ulrich T (1993). Isolation and characterization of cDNAs encoding wheat 3-hydroxy-3methylglutaryl coenzyme A reductase. Plant Physiol 102:623-628.

Baumann K, De Paolisa A, Costantino P, Gualberti G (1999). The DNA binding site of the Dof protein NtBBF1 is essential for tissuespecific and auxin-regulated expression of the rolB oncogene in plants. Plant Cell 11:323-334.

Buhaescu I, Izzedine H (2007). Mevalonate pathway: a review of clinical and therapeutical implications. Clin Biochem 40:575-584.

Cai R,Xu F, Chen L, Cheng S (2007). Modification of total RNA isolation method from different Ginkgo biloba organs. Biotechnology 17(4):38-41 (in Chinese with English abstract).

Cao X, Zong Z, Ju X, Sun Y, Dai C, Liu Q, Jiang J (2010). Molecular cloning, characterization and function analysis of the gene encoding HMG-CoA reductase from Euphorbia pekinensis Rupr. Mol Biol Rep 37:1559-1567.

Chan CS, Guo L, Shih MC (2001). Promoter analysis of the nuclear gene encoding the chloroplast glyceraldehyde-3-phosphate dehydrogenase B subunit of Arabidopsis thaliana. Plant Mol Biol 46:131-141.

Choi D, Bostock RM, Avdiushko S, Hildebrand DF (1994). Lipidderived signals that discriminate wound-and pathogen-responsive isoprenoid pathways in plants: methyl jasmonate and the fungal elicitor arachidonic acid induce different 3-hydroxy-3methylglutaryl-coenzyme A reductase genes and antimicrobial isoprenoids in Solanum tuberosum L. Proc Natl Acad Sci USA 91:2329-2333.

Dai Z, Cui G, Zhou SF, Zhang X, Huang L (2011). Cloning and characterization of a novel 3-hydroxy-3-methylglutaryl coenzyme A reductase gene from Salvia miltiorrbiza involved in diterpenoid tanshinone accumulation.J Plant Physiol 168:148-157.

Edwards D, Murray JAH, Smith AG (1998). Multiple Genes Encoding the Conserved CCAAT-Box Transcription Factor Complex Are Expressed in Arabidopsis. Plant Physiol 117:1015-1022.

Elmayan T, Tepfer M (1995). Evaluation in tobacco of the organ specificity and strength of the rol D promoter, domain $A$ of the $35 \mathrm{~S}$ promoter and the 35S-2 promoter. Transgenic Res 4:388-396.

Eulgem T, Rushton PJ, Robatzek S, Somssich IE (2000). The WRKY superfamily of plant transcription factors. Trends Plant Sci 5:199206.

Fey V, Wagner R, Brautigam K, Wirtz M, Hell R, Dietzmann A, Leister D, Oelmüller R, Pfannschmidt T (2005). Retrograde plastid redox signals in the expression of nuclear genes for chloroplast proteins of Arabidopsis thaliana.J Biol Chem 280:5318-5328.

Fuglevand G, Jackson JA, Jenkins GI (1996). UV-B, UV-A, and blue light signal transduction pathways interact synergistically to regulate chalcone synthase gene expression in Arabidopsis. Plant Cell 8:23472357.

Gao S, Lin J, Deng Z, Li Y, Sun X, Tang K (2006). Molecular cloning, characterization and functional analysis of a 2C-methyl ${ }_{D}$-erythritol 2,4-cyclodiphosphate synthase gene from Ginkgo biloba.J Biochem Mol Biol 39:502-510.

Gertz HJ, Kiefer M (2004). Review about Ginkgo biloba special extract EGb 761 (Ginkgo). Curr Pharm Design 10:261-264.

Gong Y, Liao Z, Chen M, Zuo K, Guo L, Tan Q, Huang Z, Kai G, Sun X, Tan F, Tang K (2005). Molecular cloning and characterization of a 1-deoxy-D-xylulose 5-phosphate reductoisomerase gene from Ginkgo biloba. DNA Seq 16:111-120.

Gong YF, Liao ZH, Guo BH, Sun XF, Tang KX (2006).Molecular cloning and expression profile analysis of Ginkgo biloba DXS gene encoding 1-deoxy-D-xylulose 5-phosphate synthase, the first committed enzyme of the 2-C-methyl-D-erythritol 4phosphate pathway. Planta Med 72:329-335.

Gubler F, Raventos D, Keys M, Watts R, Mundy J, Jacobsen JV (1999). Target genes and regulatory domains of the GAMYB transcriptional activator in cereal aleurone. Plant J 17:1-9.

Hannah MA, Heyer AG, Hincha DK (2005). A global survey of gene regulation during cold acclimation in Arabidopsis thaliana. PLoS Genet 1:e26s.

Hartmann U, Sagasser M, Mehrtens F, Stracke R, Weisshaar B (2005). Differential combinatorial interactions of cis-acting elements 
recognized by R2R3-MYB, BZIP, and BHLH factors control lightresponsive and tissue-specific activation of phenylpropanoid biosynthesis genes. Plant Mol Biol 57:155-171.

Hemm MR, Rider SD, Ogas J, Murry DJ, Chapple C (2004). Light induces phenylpropanoid metabolism in Arabidopsis roots. Plant J 38:765-778.

Heyworth CJ, Iason GR, Temperton V, Jarvis PG, Duncan AJ (1998). The effect of elevated $\mathrm{CO}_{2}$ concentration and nutrient supply on carbon based plant secondary metabolites in Pinus sylvestris L. Oecologia 115:344-350.

Hunter WN (2007). The non-mevalonate pathway of isoprenoid precursor biosynthesis. J Biol Chem 282:21573-21577.

Kang MK, Nargis S, Kim SM, Kim SU (2013). Distinct expression patterns of two Ginkgo biloba 1-hydroxy-2-methyl-2-(E)-butenyl4-diphosphate reductase/isopentenyl diphosphate synthase (HDR/IDS) promoters in Arabidopsis model. Plant Physiol Biochem 62:47-53.

Kaplan B, Davydov O, Knight H, Galon Y, Knight MR, Fluhr R, Fromm H (2006). Rapid transcriptome changes induced by cytosolic $\mathrm{Ca}^{2+}$ transients reveal ABRE-related sequences as $\mathrm{Ca}^{2+}$ responsive cis-elements in Arabidopsis. Plant Cell 18:2733-2748.

Kato N, Dubouzet E, Kokabu Y, Yoshida S, Taniguchi Y, Dubouzet JG, Sato F, Yazaki K (2007). Identification of a WRKY protein as a transcriptional regulator of benzylisoquinoline alkaloid biosynthesis in Coptisjaponica. Plant Cell Physiol 48:8-18.

Kawoosa T, Singh H, Kumar A, Sharma SK, Devi K, Dutt S, Vats SK, Sharma M, Ahuja PS, Kumar S (2010). Light and temperature regulated terpene biosynthesis: hepatoprotective monoterpene picroside accumulation in Picrorbiza kurrooa. Funct Integr Genomic 10:393-404.

Kim SM, Kuzuyama T, Chang YJ, Song KS, Kim SU (2006a). Identification of class 2 1-deoxy-D-xylulose 5-phosphate synthase and 1-deoxy-D-xylulose 5-phosphate reductoisomerase genes from Ginkgo biloba and their transcription in embryo culture with respect to ginkgolide biosynthesis. Planta Med 72:234-240.

Kim SM, Kuzuyama T, Chang YJ, Kwon HJ, Kim SU (2006b). Cloning and functional characterization of 2-C-methyl-Derythritiol 4-phosphate cytidyltransferase (GbMECT) gene from Ginkgo biloba. Phytochemistry 67:1435-1441.

Kim SM, Kuzuyama T, Chang YJ, Kim SU (2006c). Cloning and characterization of 2-C-methyl-D-erythritiol 2,4-cyclodiphosphate synthase (MECS) gene from Ginkgo biloba. Plant Cell Rep 25:829935.

Kim SM, Kuzuyama T, Kobayahsi A, Sando T, Chang YJ, Kim SU (2008a). 1-Hydroxy-2-methyl-2-(E)-butenyl 4-diphosphate reductase (IDS) is encoded by multicopy genes in gymnosperms Ginkgo biloba and Pinus taeda. Planta 227:287-298.

Kim SM, Kim YB, Kuzuyama T, Kim SU (2008b). Two copies of $4-$ (cytidine 5'-diphospho)-2-C-methyl-D-erythritol kinase (CMK) gene in Ginkgo biloba: molecular cloning and functional characterization. Planta 228:941-950.

Kim SM, Kim SU (2010). Characterization of 1-hydroxy-2-methyl-2(E)-butenyl-4-diphosphate synthase (HDS) gene from Ginkgo biloba. Mol Biol Rep 37:973-979.

Kim JH, Lee KI, Chang YJ, Kim SU (2012). Developmental pattern of Ginkgo biloba levopimaradiene synthase (GbLPS) as probed by promoter analysis in Arabidopsis thaliana. Plant Cell Rep 31:11191127.

Koornneef A, Leon-Reyes A, Ritsema T, Verhage A, Den Otter FC, Van Loon L, Pieterse CMJ (2008). Kinetics of salicylate-mediated suppression of jasmonate signaling reveal a role for redox modulation. Plant Physiol 147:1358-1368.

Korth KL, Jaggard DAW, Dixon RA (2000). Developmental and lightregulated post-translational control of 3-hydroxy-3-methylglutarylCoA reductase levels in potato. Plant J 23:507-516.

Learned RM, Connolly EL (1997). Light modulates the spatial patterns of 3-hydroxy-3-methylglutaryl coenzyme A reductase gene expression in Arabidopsis thaliana. Plant J 11:499-511.

Liao H-J, Zheng Y-F, Li H-Y, Peng G-P (2011). Two new ginkgolides from the leaves of Ginkgo biloba. Planta Medica 77:1818-1821.

Liao Z, Tan Q, Chai Y, Zuo K, Chen M, Gong Y, Wang P, Pi Y, Tan F, Sun X, Tang K (2004). Cloning and characterisation of the gene encoding HMG-CoA reductase from Taxus media and its functional identification in yeast. Funct Plant Biol 31:73-81.

Liao P, Zhou W, Zhang L, Wang J, Yan X, Zhang Y, Zhang R, Li L, Zhou G, Kai G (2009). Molecular cloning, characterization and expression analysis of a new gene encoding 3-hydroxy-3methylglutaryl coenzyme A reductase from Salvia miltiorrbiza. Acta Physiol Plant 31(3):565-572.

Liao Y, Xu F, Zhu J, Wang Y, Cheng S (2008). Separation and determination of terpene trilactiones by gas chromatography with wide bore capillary column. Acta Agric Borealioccidentalis Sinica 17(1):146-149 (in Chinese with English abstract).

Lichtenthaler H, Rohmer M, Schwender J (1997). Two independent biochemical pathways for isopentenyl diphosphate and isoprenoid biosynthesis in higher plants. Physiol Plant 101:643-652.

Ma DM, Pu GB, Lei CY, Ma LQ, Wang HH, Guo YW, Chen JL, Du ZG, Wang H, Li GF, Ye HC, Liu BY (2009). Isolation and characterization of AaWRKY1, an Artemisia annua transcription factor that regulates the Amorpha-4, 11-diene synthase gene, a key gene of artemisinin biosynthesis. Plant Cell Physiol 50:2146-2161.

Maldonado-Mendoza IE, Burnett RJ, Nessler CL (1992) Nucleotide sequence of a cDNA encoding 3-hydroxy-3-methylglutaryl 3Hydroxy-3-methylglutaryl coenzyme A reductase from Catharanthus roseus. Plant Physiol 100:1613-1614.

Martin DM, Gershenzon J, Bohlmann J (2003). Induction of volatile terpene biosynthesis and diurnal emission by methyl jasmonate in foliage of Norway spruce. Plant Physiol 132:1586-1599.

Menke FL, ParchmannS, Mueller MJ, Kijne JW, Memelink J (1999). Involvement of the octadecanoid pathway and protein phosphorylation in fungal elicitor-induced expression of terpenoid indole alkaloid biosynthelic gene in Catharanthus roseus. Plant Physiol 119:1289-1296.

Mohanta TK, Tamboli Y, Zubaidha P (2014). Phytochemical and medicinal importance of Ginkgo biloba L. Nat Prod Res 28:746752. 
34

Mosaleeyanon K, Zobayed SMA, Afreen F, Kozai T (2005). Relationships between net photosynthetic rate and secondary metabolite contents in St. John's wort. Plant Sci 169:523-531.

Nakano T, Suzuki K, Ohtsuki N, Tsujimoto Y, Fujimura T, Shinshi H (2006). Identification of genes of the plant-specific transcriptionfactor families cooperatively regulated by ethylene and jasmonate in Arabidopsis thaliana.J Plant Res 119:407-413.

Pang YZ, Shen GA, Berges T, Cardier H, Wu WS, Sun XF, Tang KX (2006). Molecular cloning, characterization and heterologous expression in Saccharomyces cerevisiae of a mevalonate diphosphate decarboxylase cDNA from Ginkgo biloba. Physiol Plant 127:19-27.

Pauw B, Hilliou FA, Martin VS, Chatel G, de Wolf CJ, Champion A, Pré M, van Duijn B, Kijne JW, van der Fits L, Memelink J (2004). Zinc finger proteins act as transcriptional repressors of alkaloid biosynthesis genes in Catharanthus roseus.J Biol Chem 279:5294052948.

Pu GB, Ma DM, Chen JL, Ma LQ, Wang H, Li GF, Ye HC, Liu BY (2009). Salicylic acid activates artemisinin biosynthesis in Artemisia annua L. Plant Cell Rep 28:1127-1135.

Robert-Seilaniantz A, Grant M, Jones JDG (2011). Hormone crosstalk in plant disease and defense: more than just jasmonate-salicylate antagonism. Annu Rev Phytopathol 49:317-343.

Sakai H, Aoyama T, Oka A (2000). Arabidopsis ARR1 and ARR2 response regulators operate as transcriptional activators. Plant J 24:703-711.

Schwarz KM (1994). Terpen-biosynthese in Ginkgo biloba: Eine Uberraschende geschichte. Ph.D thesis, Eidgenossischen Technischen Hochschule, Zurich, Switzerland.

Schwarz M, Arigoni D (1999). Ginkgolide biosynthesis. In: Cane D (Ed). Comprehensive Natural Products Chemistry. Pergamon Press, Oxford, vol. 2, pp 367-400.

Shen GA, Pang YZ, Wu WS, Liao ZH, Zhao LX, Sun XF, Tang KX (2006). Cloning and characterization of a root-specific expressing gene encoding 3-hydroxy-3-methylglutaryl coenzyme A reductase from Ginkgo biloba. Mol Biol Rep 33:117-127.

Singh B, Kaur P, Singh RD, Ahuja PS (2008). Biology and chemistry of Ginkgo biloba. Fitoterapia 79: 401-418.

Singh H, Gahlan P, Kumar S (2013). Cloning and expression analysis of ten genes associated with picrosides biosynthesis in Picrorbiza kurrooa. Gene 515:320-328.

Smale ST, Kadonaga JT (2003). The RNA polymerase II core promoter. Annu Rev Biochem 72:449-479.

Soitamo AJ, Piippo M, Allahverdiyeva Y, Battchikova N, Aro EM (2008). Light has a specific role in modulating Arabidopsis gene expression at low temperature. BMC Plant Biol 8:13.

van Beek TA, Montoro P (2009). Chemical analysis and quality control of Ginkgo biloba leaves, extracts and phytopharmaceuticals. J Chromatogr A 1216:2002-2032.

Wang QJ, Zheng LP, Zhao PF, Zhao YL, Wang JW (2014). Cloning and characterization of an elicitor-responsive gene encoding 3hydroxy-3-methylglutaryl coenzyme A reductase involved in 20hydroxyecdysone production in cell cultures of Cyanotis arachnoidea. Plant Physiol Biochem 84:1-9.
Wang Y, Guo B, Zhang F, Yao H, Miao Z, Tang K (2007). Molecular cloning and functional analysis of the gene encoding 3-hydroxy-3methylglutaryl coenzyme A reductase from hazel (Corylus avellana L. Gasaway).J Biochem Mol Biol 40:861-869.

Xu F, Cai R, Cheng S, Du H, Wang Y, Cheng S (2008a). Molecular cloning, characterization and expression of phenylalanine ammonialyase gene from Ginkgo biloba. Afr J Biotechnol 7:721-729.

Xu F, Cheng H, Cai R, Li LL, Chang J, Zhu J, Zhang FX, Chen LJ, Wang Y, Cheng SH, Cheng SY (2008b). Molecular cloning and function analysis of an anthocyanidin synthase gene from Ginkgo biloba, and its expression in abiotic stress responses. Mol Cells 26:536-547.

Xu F, Zhang WW, Sun NN, Li LL, Cheng SY, Wang Y (2011). Effects of chlorocholine chloride on photosynthesis metabolism and terpene trilactones biosynthesis in the leaf of Ginkgo biloba. Acta Hortic Sinica 38:2253-2260.

Xu F, Li L, Zhang W, Cheng H, Sun N, Cheng S, Wang Y (2012). Isolation, characterization, and function analysis of a flavonol synthase gene from Ginkgo biloba. Mol Biol Rep 39:2285-2296.

Xu F, Huang XH, Li LL, Deng G, Cheng H, Rong XF, Li JB, Cheng SY (2013). Molecular cloning and characterization of GbDXS and GbGGPPS gene promoters from Ginkgo biloba. Genet Mol Res 12:293-301.

Xu N, Hagen G, Guilfoyle T (1997). Multiple auxin response modules in the soybean SAUR 15A promoter. Plant Sci 126:193-201.

Xu YH, Wang JW, Wang S, Wang JY, Chen XY (2004). Characterization of GaWRKY1, a cotton transcription factor that regulates the sesquiterpene synthase gene (+)-delta-cadinene synthase-A. Plant Physiol 135:507-515.

Xue GP (2003). The DNA-binding activity of an AP2 transcriptional activator $\mathrm{HvCBF} 2$ involved in regulation of low-temperature responsive genes in barley is modulated by temperature. Plant $\mathrm{J}$ 33:373-383.

Yanagisawa S, Schmidt RJ (1999). Diversity and similarity among recognition sequences of Dof transcription factors. Plant J 17:209214.

Yanagisawa S (2004). Dof domain proteins: plant-specific transcription factors associated with diverse phenomena unique to plants. Plant Cell Physiol 45:386-391.

Zeng Z, Zhu J, Chen L, Wen W, Yu R (2013). Biosynthesis pathways of ginkgolides. Pharmacogn Rev 7(13):47-52.

Zhang W, Qing G, Cheng S, Xu F (2011). Preliminary study on induction of Ginkgo callus. J Yangtze Univ (Nat Sci Edit) 8:237-239 (in Chinese with English abstract).

Zhou DX (1999). Regulatory mechanism of plant gene transcription by GT-elements and GT-factors. Trends Plant Sci 4:210-216. 